\title{
Camelots sénégalais à Buenos Aires : une négociation cosmopolite du trottoir
}

Senegalese Street Vendors in Buenos Aires: A Cosmopolitan Trading on

Sidewalks

Los vendedores ambulantes senegaleses en Buenos Aires: una negociación

cosmopolita de la acera

\section{Régis Minvielle}

\section{OpenEdition \\ Journals}

\section{Édition électronique}

URL : https://journals.openedition.org/remi/7823

DOI : $10.4000 /$ remi.7823

ISSN : $1777-5418$

\section{Éditeur}

Université de Poitiers

\section{Édition imprimée}

Date de publication : 1 septembre 2016

Pagination : 121-144

ISBN : 979-10-90426-27-6

ISSN : 0765-0752

\section{Référence électronique}

Régis Minvielle, "Camelots sénégalais à Buenos Aires : une négociation cosmopolite du trottoir », Revue européenne des migrations internationales [En ligne], vol. 32 - n² | 2016, mis en ligne le 01 septembre 2018, consulté le 14 avril 2022. URL : http://journals.openedition.org/remi/7823 ; DOI : https://doi.org/10.4000/remi.7823 


\section{Camelots sénégalais à Buenos Aires : une négociation cosmopolite du trottoir}

\section{Régis Minvielle ${ }^{1}$}

Depuis le début des années 1990, mais surtout durant la dernière décennie, l'Europe s'est progressivement muée en une véritable forteresse pour les aspirants à la migration. Les routes qui conduisent vers l'Europe se ferment sous l'effet de politiques qui laissent de moins en moins de possibilités aux migrants d'y entrer. Le pont entre les deux rives de la Méditerranée est coupé (Choplin, 2010) et les migrants subsahariens qui s'aventurent vers le nord du continent africain se retrouvent pour la plupart d'entre eux dans un état $\mathrm{d}^{\prime}$ " assignation territoriale " (Timera, 2009). Dans ces conditions, il ne reste guère de choix pour les migrants : attendre au seuil de la porte européenne en espérant qu'elle s'ouvre à nouveau, tenter de desserrer l'étreinte en se lançant dans une aventure très périlleuse à bord d'embarcations de fortune depuis les côtes lybienne, mauritanienne ou sénégalaise (Ba et Ndiaye, 2008), retourner au pays d'origine en courant le risque de devenir un paria, ou enfin imaginer des routes de rechange. C'est cette dernière option qui est retenue par des migrants africains bloqués en Lybie et qui décident de donner un nouvel élan à leurs parcours en rejoignant dans les années 1990 l'Amérique latine et plus particulièrement l'Argentine.

Cette nouvelle destination symbolise les recompositions des routes migratoires qui prennent désormais un caractère multipolaire et éclaté. Alors que les vieux couples migratoires fondés sur des liens historiques entre pays de départ et pays d'accueil (Mexique/États-Unis d'Amérique, Maghreb/France, Inde et Pakistan/Royaume-Uni), tendent à décliner, de nouvelles circulations émergent entre les Suds : La Chine qui accueille des Africains subsahariens ou encore les pays du golfe persique, qui recourent à une main-d'œuvre du Sud-est asiatique, témoignent de cette redistribution des cartes migratoires. Certes, les migrations Sud-Sud sont anciennes, mais elles reposaient essentiellement sur des systèmes d'échanges régionaux. Aujourd'hui, elles deviennent multidirectionnelles, intercontinentales et incarnent au mieux la globalisation des Suds.

1 Post-doctorant à I'Université de São Paulo, Fundação de Amparo à Pesquisa do Estado de São Paulo; minviellergis@yahoo.fr 
Bien que peu significative au regard des flux régionaux, la migration africaine en Argentine se singularise toutefois par sa visibilité. Estimés actuellement à 5000 individus, dont plus de 3000 Sénégalais (Maffia et al., 2012), les Africains occupent pour la majorité d'entre eux le créneau de la vente ambulante sur les artères, les places et les marchés de Buenos Aires. Monnet, Giglia et Capron (2007) ont élaboré une typologie des vendeurs ambulants : ceux qui possèdent un kiosque, ceux qui effectuent leur commerce à partir d'un stand démontable, ceux qui utilisent un charriot ou autre type de support à roulettes, ceux qui circulent sur les voies entre les véhicules et ceux qui opèrent sur les trottoirs. Dans cette contribution, $c^{\prime}$ est surtout cette dernière catégorie dont il sera question à travers l'examen de l'inscription socio-spatiale des Sénégalais aux côtés d'autres communautés africaines (Guinéens, Nigérians, Ghanéens, Sierra Léonais et Libériens), mais aussi latino-américaines (Péruviens, Paraguayens et Boliviens) dans le quartier central d'Once. L'objectif, ici, est de rendre compte des pratiques qui se jouent dans l'espace public et des liens sociaux et cosmopolites qui se tissent autour de la vente ambulante. Au réseau migratoire du proche, c'est-à-dire en lien avec la société d'origine, se superposent d'autres formes de solidarités produites par l'expérience de la rue.

Nos travaux s'inscrivent dans une démarche socio-anthropologique qui a pour particularité d'étudier l'activité sociale dans une perspective d'interaction. II s'agit d'appréhender les échanges sociaux, les rapports inter-ethniques, non pas dans une opposition binaire de type structuraliste, mais dans une perspective dynamique. Examiner le commerce ambulant selon cette approche revient à observer des processus, des continuités et des ruptures à partir d'un ensemble de liens, mais aussi de conflits qui s'établissent entre les différents acteurs, entre ceux-ci et les nouveaux espaces qu'ils investissent. Afin de répondre à cet objectif, l'ethnographie a constitué la méthode d'enquête privilégiée. En se penchant sur l'ordinaire, l'immersion permet de comprendre les cultures de la rue en montrant "ce qu'est sa normalité sans gommer ses particularités " (Geerz, 1998 : 86). Imparfaite en raison de son niveau d'approximation et d'incertitude, l'ethnographie reste toutefois une valeur sûre à I'heure d'appréhender les pratiques non officielles qui échappent à tout dispositif réglementaire, celles qu'on ne peut pas saisir dans un questionnaire ou dans une interview. Le monde de la rue fait souvent écho à celui de la débrouille qui se glisse dans les failles des dispositifs de réglementation étatique.

L'immersion permet alors de mettre en évidence à la fois des situations ou des conduites qui ne sont pas dicibles, soit parce qu'elles ont un caractère occulte, soit parce qu'elles sont routinières et jugées peu pertinentes par les acteurs. En parallèle à ce contexte d'observation, nous avons recueilli des données de type biographique. L'objectif était d'identifier et de reconstituer les différents itinéraires sociaux ou les différentes étapes qui jalonnent la vie de chacun, du pays d'origine jusqu'au pays d'arrivée ${ }^{2}$.

2 Cette enquête, ponctuée de deux séjours de terrain à un an d'intervalle en 2010 et en 2011 et d'une durée totale de sept mois, s'est inscrite dans le cadre d'un doctorat qui avait pour objectif d'ausculter les contours de ce dispositif migratoire sud-sud (Minvielle, 2013). Cette thèse était par ailleurs rattachée au programme ANR MIGRELI (Instances religieuses et d'origine confessionnelle sur les routes de la migration africaine) coordonné par Sophie Bava, Institut IRD, 2009-2012. 
Avant d'évoquer ces inscriptions spatiales et les rapports sociaux qui s'élaborent dans la rue, nous présenterons une brève genèse de l'arrivée des migrants africains sur les bords du Rio de la Plata.

\section{De la figure de l'esclave au vendeur ambulant}

C'est d'abord dans le cadre du commerce d'esclaves, que les premiers Africains rejoignent contre leur gré, les côtes argentines à partir du XVle siècle. II faut ensuite attendre la fin du XIXe siècle, et selon une tout autre logique migratoire, pour voir des Capverdiens s'installer dans les quartiers portuaires de Buenos Aires. Ces derniers s'embarquaient à bord de baleiniers britanniques qui, avant de mettre le cap vers la Géorgie du Sud, faisaient escale au Cap-Vert pour se ravitailler en charbon. Au terme de ces campagnes de pêche, certains décidèrent de s'installer dans les quartiers portuaires de Buenos Aires. Un deuxième flux migratoire se produit entre 1920 et 1933, puis un troisième après 1946, avant de décroître progressivement et de s'éteindre définitivement au début des années 1960 en lien avec le déclin des activités maritimes (Contarino Sparta, 1998) Dans cette seconde moitié du XXe siècle, I'histoire des mobilités africaines à l'échelon du sous-continent latino-américain concerne surtout les étudiants ${ }^{3}$.

Puis à partir des années 1990, c'est au tour de ressortissants Maliens résidant en Lybie et désirant échapper à l'étau méditerranéen, de tenter l'aventure transatlantique sud-sud. Lorsque l'impasse se fait trop pressante, la linéarité géographique du parcours est secondaire : ce qui compte, c'est de décrocher un nouveau point de chute. En obtenant des visas auprès de l'ambassade du Brésil à Tripoli, ces aventuriers de la migration (Bredeloup, 2008) prennent pour la première fois leur distance avec le continent africain. Dans ce voyage au long cours, si les États-Unis incarnent l'objectif final, le Brésil est d'abord envisagé comme un détour utile en vue de reconstituer un capital économique bien entamé par le coût du transport aérien. À cette époque, l'Argentine en a terminé avec la dictature militaire (1976-1983) et la transition démocratique s'accompagne d'un virage libéral qui, avant de conduire à la faillite du pays en 2001, occasionne un cycle de croissance et un boom de l'immobilier (développement des quartiers résidentiels selon le modèle nord-américain gated community). Informés de ces opportunités économiques, les Maliens, habitués aux travaux de construction depuis leur séjour en Libye, n'hésitent pas à franchir la frontière avec le pays voisin pour être embauchés dans une filière en plein essor.

Dans le même mouvement, des Sénégalais également rompus à l'expérience migratoire arrivent en ordre dispersé dans la capitale argentine à la suite de détours ou de rencontres. Alassane, casamançais d'origine, entre dans cette catégorie en décidant de rejoindre l'Argentine sur les recommandations d'un

3 Il est intéressant de souligner le rôle majeur de Cuba dans les mobilités étudiantes africaines avec l'accueil de 29106 étudiants entre 1961 et 2002. Par ailleurs, à partir des années 1980, le Brésil lance un programme de bourses destinées prioritairement aux Africains lusophones. Ce sont quelque 2140 étudiants en provenance du Mozambique et de l'Angola qui rejoignent les universités brésiliennes dans le cadre de ces partenariats ; cf. le rapport des Nations Unies (2004) South-south cooperation in support of the new partnership for Africa's development: Experiences of Africa-Latin America and the Caribbean. 
marin guinéen, de retour à Dakar après avoir fait escale à Buenos Aires à bord d'un cargo. Le récit du marin comparant Buenos Aires à une ville " européenne ", riche et qui offre des salaires en dollars ${ }^{4}$, captive Alassane qui ose franchir le pas. À son arrivée en 1996, il empile les petits boulots comme manœuvre dans le bâtiment, laveur de voitures ou encore jardinier avant d'être engagé par l'usine de montage automobile Ford en tant que carrossier-tôlier, métier qu'il avait déjà exercé auparavant en Arabie saoudite. La trajectoire ascendante se matérialise à travers des retours réguliers au Sénégal, I'acquisition d'une maison dans la banlieue de Buenos Aires et par l'ouverture en 2008 d'une boutique de babioles africaines qui lui permet de diversifier ses sources de revenus. À l'image des Maliens dans le bâtiment, le parcours d'Alassane réactualise la figure du travailleur immigré dans le secteur industriel à l'heure où le post-fordisme favorise l'expansion de l'économie informelle.

Le parcours de Leyti s'inscrit plutôt dans une dynamique commerciale. Originaire de Diourbel, il décide de retrouver en 1996 sa fiancée argentine rencontrée quelques mois auparavant à Dakar. À la différence d'Alassane qui, pour le moment, constitue seulement la tête de pont d'une migration familiale, celui qui est devenu le propriétaire de plusieurs boutiques de vêtements afro, entraîne dans son sillage d'autres compatriotes qui dépassent amplement le cadre familial pour s'étendre au réseau confrérique mouride.

Ces premières arrivées éparses et audacieuses doivent toutefois être resituées à l'aune des transformations des politiques d'accueil amorcées à I'orée des années 1990. Avec la fin des régimes autoritaires en Amérique du Sud naissent des récits multiculturels et des mesures qui favorisent la circulation des personnes (Minvielle, 2015). Par ailleurs, le Brésil, en vue de consolider son statut au sein des BRICS, opère des rapprochements économique et diplomatique avec le continent africain, symbolisés notamment par l'extension de son réseau d'ambassade et une progression constante de ses investissements. Ce volontarisme rencontre un écho favorable en la personne du président sénégalais Abdoulaye Wade soucieux de réorienter la politique étrangère vers les pays émergents, comme la Chine, I'Inde et le Brésil (Colom-Jaén, 2012). Ces rapprochements aboutiront en 2005 à la signature d'accords de coopération dans les domaines de la culture, des télécommunications et dans l'attribution de visas. Sous l'effet conjugué de ces nouveaux partenariats et de la fermeture de l'ambassade d'Argentine à Dakar en $2002^{5}$, le Brésil va désormais constituer une porte d'entrée ${ }^{6}$ pour une nouvelle génération de migrants. Si les pionniers de la migration africaine en Argentine arrivent en ordre dispersé, disposent de qualifications et sont généralement déjà coutumiers de l'expérience migratoire, le profil de leurs successeurs diffère sensiblement. À l'image de l'émigration

\footnotetext{
4 Lorsque le peso remplace I'austral en 1992, une loi de convertibilité impulsée par une politique de réévaluation aligne le peso à hauteur du dollar. Ce n'est qu'avec la crise bancaire et monétaire de 2002 que le peso commencera à décroître sérieusement. Aujourd'hui, un dollar équivaut à environ neuf pesos.

5 Conséquence directe de la crise financière de 2001 qui contraint l'État argentin à suivre un plan d'austérité.

6 Après avoir obtenu un visa touristique auprès du consulat du Brésil à Dakar, les néomigrants ont recours à des passeurs locaux pour franchir clandestinement la frontière entre le Brésil et l'Argentine. Pour des explications plus détaillées concernant les modalités de passage, voir Minvielle (2015).
} 
sénégalaise en Italie (Schmidt di Friedberg, 1993), à chaque temporalité des flux correspondent de nouvelles figures. D'origine rurale en provenance principalement du bassin arachidier autour des villes de Diourbel, de Touba, de Thiès, de Kaolack, les nouveaux arrivants des années 2000 sont pour l'essentiel des jeunes hommes célibataires (entre vingt et trente ans) peu instruits ${ }^{7}$. Ils appartiennent majoritairement à la confrérie mouride et participent ainsi de la reproduction d'un " idéal-type " migratoire, fondé sur le commerce et déjà éprouvé dans les grandes villes d'Afrique (Copans, 1980) d'Europe occidentale (Bava, 2005 ; Riccio, 2006) ou encore des États-Unis d'Amérique (Ebin et Lake, 1992).

Le mouridisme est une organisation soufie qui est apparue à la fin du XIXe siècle et qui s'organise autour de relations verticales entre les marabouts (Cheikh) et leurs disciples (Talibés). La vie communautaire, particulièrement intense, est une dimension très importante. Fondée par Cheikh Amadou Bamba, cette doctrine établit comme principes fondamentaux l'assiduité au travail et l'éthique de la solidarité. Jusqu'au début des années 1970, la communauté mouride est essentiellement rurale mais, suite aux sécheresses successives des années 1968, 1970 et 1972 et à la chute du cours de l'arachide, les mourides se lancent à grande échelle dans le commerce, en premier lieu à Dakar, autour du marché Sandaga, puis dans les villes de migrations africaines et, enfin, dans les villes d'Europe et d'Amérique du Nord (Copans, 2010). Certains de ces talibés empruntent les chemins de la migration internationale et tissent à travers leur éparpillement dans le monde, des liens commerciaux grâce, en partie, à la figure du marabout qui, par son autorité, joue un rôle d'intermédiation entre les disciples.

\section{La mondialisation de la vente à la sauvette}

À l'image de nombreuses métropoles, Buenos Aires affronte un processus de désindustrialisation propice au développement d'activités informelles qui agencent désormais un pan entier de l'économie autour de transactions et d'emplois échappant au contrôle et à la régulation. En première ligne de ces activités, le commerce ambulant et ses camelots qui déploient leurs marchandises sur les places, sur les marchés ou encore aux abords des gares. Avec ses gains incertains, cette activité illustre cette généralisation de la flexibilisation et l'accroissement de la précarisation. Peu institutionnalisée et aux frontières poreuses entre légal et illégal, entre formel et informel (Telles, 2009), la vente de rue constitue souvent l'unique ressource pour des migrants ou des autochtones exclus d'un marché du travail de plus en plus spécialisé. Bien qu'elle échappe aux réglementations, la vente de rue n'est pourtant pas reléguée en périphérie ou dans les marges urbaines. Au contraire, elle s'inscrit souvent au cœur des villes, là où les espaces de flux et de croisements favorisent les implantations marchandes. Ces espaces sont généralement situés à proximité des nœuds urbains où s'incrustent des gares ferroviaires ou routières, des stations de métro principales ou encore des axes ou carrefours routiers.

7 La majorité d'entre eux ont reçu comme seuls enseignements, l'étude du coran dispensée par les Daara (mot qui signifie " maison " en arabe). Les enfants consacrent une partie du temps à étudier les principes de la religion islamique et le reste aux travaux agricoles. 
Ce sont ces lieux de transport que les vendeurs de rue investissent en priorité et qui se surajoutent à de nombreux commerçants dits établis. Comme le soulignent Lombard et Steck (2004), ces lieux qui se caractérisent par la " multimodalité " de moyens de transport, favorisent le déploiement d'activités multiples destinées à répondre aux besoins des passagers. Autour de ces nœuds urbains qui accueillent des passagers en provenance d'autres lieux ou en attente d'un départ, ou en transit, se greffent ainsi une pluralité de commerces : des brasseries, des hôtels, des kiosques à journaux, des téléboutiques, mais aussi des galeries marchandes de prêt-à-porter qui ne constituent qu'un échantillon de la multiplicité des services aux voyageurs.

La vente ambulante, depuis le petit camelot à la sauvette jusqu'à la caravane spécialement aménagée pour vendre des gaufres ou des frites, en passant par les chariots des marchands de glace, cherche également à s'enraciner dans ces lieux de transit et au plus près des infrastructures de transport.

Foncièrement associé à la mobilité, le commerce de rue consiste soit à apporter un bien au client susceptible de l'acheter, soit à amener le consommateur final jusqu'à un espace qui expose les produits à vendre. Ce type de commerce est parfaitement adapté au rythme accéléré des passants qui réduisent au minimum les temps de pauses nécessaires. II présente cet avantage d'intercepter des individus en mouvement. Les termes utilisés pour décrire la profession, tels que " colporteur ", " marchand ambulant ", " camelot " ou encore " vendeur à la sauvette " se réfèrent directement à la mobilité.

À Buenos Aires, comme à Marseille, Paris, Milan ou Barcelone, ce sont les migrants qui installent leur marchandise sur le trottoir ou qui se déplacent à la recherche du client potentiel. Le quartier d'Once, situé dans le centre-ville est certainement le quartier qui illustre au mieux ce monde de l'informalité.

\section{Once : un espace marchand cosmopolite}

Ce qui étonne le passant lorsqu'il se rend dans le quartier d'Once, c'est d'abord la densité du commerce. Dans ce dédale de rues bordées par les grandes artères Puyrredón, Corrientes et Rivadavia, Callao (cf. Figure 1), I'offre commerciale est prolifique. Elle est principalement de petite taille et se décline sous différentes formes: petites boutiques qui ont pignon sur rue, espaces réduits agglomérés les uns aux autres dans une galerie marchande qui relie les rues entre elles. Aucune vitrine luxueuse et aucune grande surface ne viennent s'incruster dans le territoire et au regard de l'attractivité des prix, l'offre commerciale s'adresse avant tout aux classes populaires. Mais la présence de stations de métro, d'une gare ferroviaire et d'une gare routière, assure un flux continu de population très hétérogène et à même de consommer. Chaque jour, ce sont des hordes de chalands et de travailleurs qui, au gré de leurs allers et venues dans le quartier, alimentent une économie de "bazar ", entendue comme " un système singulier de relations sociales, centré autour de la production et de la consommation de biens et de services " (Geerz, 2003).

Place marchande pleinement intégrée à une mondialisation qui implique désormais toute la base de la pyramide sociale (Prahalad, 2004) incarnée par des "commerçants nomades" (Tarrius, 2002), Once fait partie de ces lieux qui relient entre eux des lieux de production, de négoce, d'entreposage de (re)vente 
au détail et de consommation. Au sein de ce système marchand transnational, s'échangent des marchandises souvent similaires, regroupées sous le terme générique de "small comodities" made in China. À Once, comme à Belsunce, à Brás (São Paulo), ou encore sur les étals du grand marché de Yiwu au sud de Shanghai (Pliez, 2012), on retrouve les mêmes bijoux-fantaisies, articles de décoration, des biens d'équipement domestiques ou encore du petit matériel électronique qui incarnent ce que Levitt prévoyait déjà en 1983 comme le " marché global des produits de consommation standardisés ".

En attirant des masses de piétons qui convergent vers les différents terminaux de transport, les avenues sont des lieux privilégiés pour l'implantation de vendeurs à la sauvette. En s'inscrivant dans les itinéraires possibles des acheteurs, ces grandes artères représentent un point de fixation qui permet le développement des échanges. À mesure que l'on se rapproche de la place Miserere qui fait face à la gare, la concentration des vendeurs de rue augmente. En s'y installant, les Sénégalais rejoignent ainsi d'autres communautés africaines (Guinéens, Nigérians, Ghanéens, Sierra Léonais et Libériens), mais aussi latino-américaines, qui inscrivent également leurs pratiques dans l'espace public : cette présence ne déroge pas à la règle qui veut que " toute l'histoire économique montre que l'étranger fait partout son apparition comme commerçant, et le commerçant comme étranger " (Simmel, 1984.).

Ils se partagent ce créneau économique selon des modalités distinctes. Les Argentins vendent des chori-pan (sandwich à base de chorizo), des empanadas (chausson à base de viande, de jambon ou de fromage), du café, des glaces, ou encore des boissons fraîches. Les Péruviens sont les plus nombreux et exposent des survêtements et tee-shirt de contrefaçon à l'effigie des marques Adidas, Nike et Lacoste, des écharpes, des bonnets, des casquettes, des chaussettes, des sous-vêtements, des chaussures, des espadrilles, ou encore des étuis de portables et des barrettes pour cheveux. À leurs côtés, des Boliviens sont spécialisés dans les fruits et légumes. Enfin, des Brésiliens circulent en portant des hamacs sur leurs épaules.

À l'image de Belsunce à Marseille, du quartier de la Porte Saint-Denis à Paris (Dinh, 2009), ou encore de Côte-des-Neiges à Montréal (Germain et Blanc, 1998), Once est également une "centralité minoritaire " (Raulin, 1988) qui a vu se succéder des strates de migrants. Ce sont d'abord les Italiens qui s'y installent, à la fin du XIXe siècle, pour établir des petits commerces. Ils sont bientôt rejoints par des Juifs d'Europe de l'Est qui fuient les pogroms, puis par des Juifs séfarades du Maroc (à partir de 1905), de Grèce, de Turquie et de Syrie. Certains d'entre eux s'installent à Once et se lancent dans le commerce de meubles et de textiles. Ils maintiennent leurs traditions culturelles en créant des synagogues, des associations, des écoles et des collèges confessionnels. Ces institutions, ainsi que l'inscription commerçante dans le quartier sont encore visibles aujourd'hui. Dans les années 1980, c'est au tour des Sud-Coréens d'investir les lieux. L'émigration sud-coréenne s'amorce dès les années 1960 ; elle est encouragée par un régime politique qui souhaite contrôler la croissance démographique (Mera, 2006). Si les premiers migrants s'installent dans les régions rurales d'Argentine pour s'insérer dans l'activité agricole, ceux qui leur succèdent s'implantent à Buenos Aires pour ouvrir des boutiques "bazars ". À l'intérieur de ces boutiques, on trouve aussi bien des produits ethniques comme 
des statuettes de Bouddha, de dragons, des objets de massage, ainsi que des objets du quotidien (papeterie ou ustensiles de cuisine) de décoration (lustres, lampes, vases, etc.) ou personnels (pendentifs ou montres).

Le découpage ethnique de l'espace marchand donne à voir au passant cette mosaïque cosmopolite. Les Juifs sont établis dans les rues Castelli, Sarmiento, Lavalle et Azcuenaga où s'alignent des boutiques où I'on peut acheter des costumes, des chemises, des robes de mariage ou encore des tissus en gros ou au détail. Les boutiques coréennes sont essentiellement situées dans les rues PasoSaavedera et Larrea.

\section{Figure 1 : Plan d'Once}

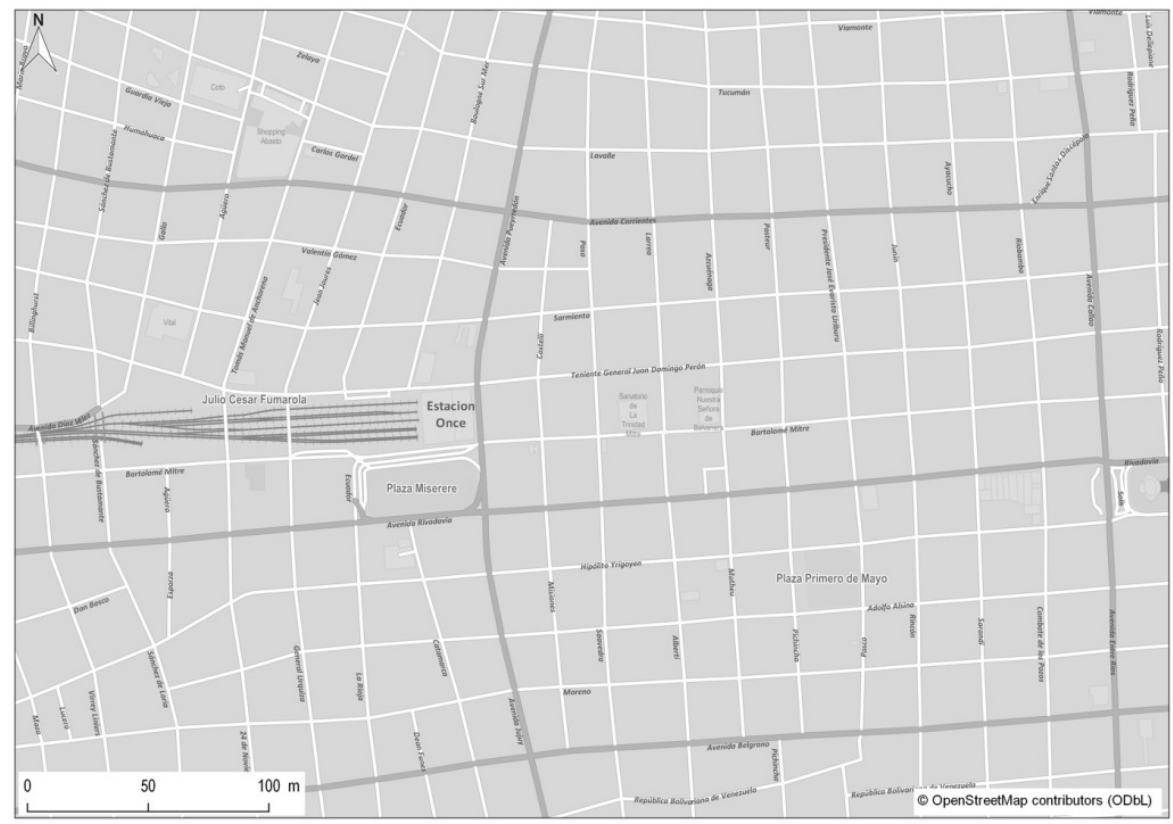

Source : Carte interactive de la municipalité de Buenos Aires ; https://mapa.buenosaires.gob.ar

La place Miserere prolonge ce cosmopolitisme avec la présence de musiciens équatoriens qui se mettent en scène avec leurs parures de plumes empruntées aux Amérindiens d'Amérique du Nord. À quelques pas des musiciens qui entonnent leur récital de musique andine, des prostituées dominicaines interpellent les passants pour proposer leurs services.

Tous ces acteurs, faute de pouvoir accéder à l'emploi salarié, envisagent alors la rue comme unique ressource. L'absence de compétences à faire valoir sur un marché du travail devenu de plus en plus spécialisé et générateur d'une compétition toujours plus acharnée entre les demandeurs d'emploi, le déficit de capital social essentiel dans la quête de l'emploi salarié, conduit de nombreux acteurs des classes populaires et de la migration à (ré) « inventer leur quotidien " (De Certeau, 1990). 
Certains ont pourtant tenté leur chance sur le marché du travail en dépit d'un déficit de qualifications, conjugué bien souvent à un statut juridique précaire ${ }^{8}$. C'est le cas d'Amadou, originaire de Guinée-Conakry, présent en Argentine depuis 2007 et qui, un an plus tard, décroche d'abord un emploi d'électricien. Le revenu étant jugé insuffisant, il s'essaie ensuite comme magasinier pour le compte d'un grand quotidien de presse nationale. II se sent exploité et affirme être victime de discrimination par rapport aux locaux qui, selon lui, sont mieux

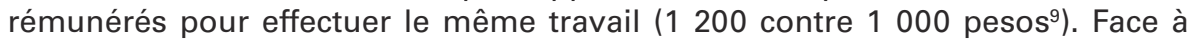
ces échecs successifs, Amadou retourne dans la rue plus rémunératrice et moins contraignante :

"Dans la rue, j'ai pas un patron qui me dit ce que je dois faire et puis surtout on gagne plus " (Amadou, conversation informelle, 18 juillet 2010).

À ses côtés, Mahamadou, son acolyte sénégalais, pointe aussi du doigt les salaires bas des emplois peu qualifiés:

"Au maximum, on peut me donner un salaire de 2000 pesos, c'est ce que je gagne avec la vente. Pour moi, ce n'est pas un problème, moi par exemple, je peux arriver dans n'importe quel endroit. Si j'ai de l'argent, c'est pas un problème, je sais me débrouiller. Nous, on est habitué à souffrir, à se coucher sans manger, à dormir mal " (Mahamadou, conversation informelle, 18 juillet 2010).

Pour la plupart des jeunes mourides, la vente de rue constitue une ressource qui, bien que contraignante et aléatoire, offre des revenus supérieurs au travail sous ou peu qualifié.

\section{Le créneau de la bijouterie de pacotille}

C'est à partir des années 2000, avec l'arrivée des Africains, que la bijouterie de pacotille fabriquée en Chine voit le jour dans les rues de Buenos Aires. Cette activité $s^{\prime}$ initie à travers I'appui de $\mathrm{Myrar}^{10}$, une association de prise en charge des réfugiés et des migrants. Spécialisée dans l'aide à l'insertion professionnelle des migrants, Myrar propose notamment des formations professionnelles dans I'auto-entreprenariat. En poursuivant ces objectifs, I'association lance en 2002 un programme de microcrédits financé par la section argentine du Haut Commissariat pour les réfugiés. Entre 2002 et 2005, Myrar octroie environ 250 crédits à des migrants issus de l'Amérique latine et de l'Afrique de l'Ouest. Cet appui financier leur permet de démarrer une activité, principalement autour de la production et de la commercialisation de produits textiles ou gastronomiques, dans des ferias ${ }^{11}$ artisanales. Ils tiennent des stands de vêtements, de beignets ou encore de bijouterie fantaisie. Si l'association permet de faire émerger un créneau économique à travers l'apport d'un capital, elle encadre et limite

8 À leur arrivée, les Africains formulent une demande de statut de réfugié en vue d'obtenir dans un premier temps un titre de séjour provisoire (permiso de residencia precaria, renommé precaria) valide pour une période de trois mois renouvelables.

9 En 2008, le salaire minimum était de 1200 pesos. Il a été augmenté en janvier 2010 à 1500 pesos.

10 Le nom complet de I'association est Migrantes y Refugiados en Argentina, que I'on peut traduire par "Migrants et Réfugiés en Argentine ".

11 Peut se traduire par "foire " ou " marché ", selon le contexte. 
toutefois I'activité au seul cadre formel et réglementé de la feria. Dans l'optique d'optimiser leurs gains, les Africains décident d'étendre leur activité au-delà de la feria en installant leurs marchandises sur les trottoirs des rues commerçantes de Buenos Aires.

Mais à partir de 2005, devant I'afflux des Sénégalais, ces associations limitent leur assistance financière. En réponse au tarissement de cette ressource, les jeunes mourides qui arrivent à Buenos Aires peuvent s'appuyer sur la solidarité confrérique pour démarrer l'activité. En arrivant dans la capitale argentine, le nouveau venu dispose d'au moins un contact parmi ses compatriotes. Cette personne ressource, qui peut être un membre de sa famille, un ami, ou un ancien voisin, se charge alors de l'accueillir dans son foyer. Les membres du foyer mettent à disposition du nouveau venu un lit et facilitent son insertion dans le commerce. Les Sénégalais du foyer organisent une collecte destinée à I'achat d'une valise et d'un stock de bijoux, en d'autres termes un prêt qui sera échelonné sur plusieurs mois avec les premiers bénéfices de la vente.

S'ils sont présents à des postes fixes comme les autres groupes d'Africains, les Sénégalais sont en revanche les seuls à pratiquer la mobilité permanente en sillonnant les rues de la capitale. Équipés d'une valise de type attaché-case, les colporteurs entrent dans les cafés, les restaurants, les boutiques ou encore dans les parcs et autres lieux publics à la recherche de clients potentiels.

Ceux qui s'établissent à un poste fixe peuvent être aussi mobiles. Certes, ils ne parcourent pas au moins une dizaine de kilomètres par jour comme les commerçants à la valise, mais ils peuvent changer d'emplacement assez régulièrement s'il n'apporte pas les gains escomptés. D'autre part, avec la fermeture des galeries marchandes et des commerces officiels le dimanche, Once se vide complètement de sa population. Les rythmes de l'organisation du travail et l'ordre commercial imposent au vendeur à la sauvette de déplacer son étal au gré des dynamiques d'occupation urbaine. En fin de semaine, les ferias prennent souvent le relai des espaces marchands traditionnels qui s'articulent le reste du temps autour des nœuds de transports et des grandes artères. Le temps libre déplace cette économie de bazar. En retrait des grands axes de circulation et du bourdonnement urbain, des parcs et des places sont envahis par des flâneurs et des familles qui recherchent souvent la quiétude. Les Africains, tout comme des artisans-commerçants, des antiquaires ou encore des bouquinistes et des disquaires, accompagnent alors ces mouvements en installant leurs objets et leurs produits dans ces espaces du temps libre qui, peu à peu, se transforment en lieux de commerce.

\section{Les liens sociaux du commerce de rue}

\section{Faire sa place}

Si, dans les ferias, les migrants ont bénéficié d'un appui institutionnel pour s'installer, il ne peut en être de même dans la rue, en raison de l'illégalité de la vente de rue. Pour les vendeurs à la sauvette, l'implantation marchande consiste bien souvent à obtenir le consentement d'autres commerçants. 
Après avoir identifié le lieu dans lequel on souhaite s'installer, il faut obtenir l'accord des autres commerçants de rue situés à proximité de l'emplacement convoité. L'accord s'obtient généralement s'il est démontré qu'on ne va pas concurrencer directement son voisin avec une marchandise similaire. L'instauration de ces frontières marchandes étanches entre les communautés permet d'éviter les tensions d'ordre économique qui sont parfois à l'origine des clivages de type ethnique.

Dans la cité phocéenne par exemple, les commerçants maghrébins ont très mal accepté l'arrivée des vendeurs de rue africains à la fin des années 1970 (Koné, 1995). Accusés de concurrence déloyale parce qu'ils vendaient les mêmes articles à un prix moins élevé, dans le même périmètre (quartier de Belsunce) que les boutiques maghrébines, les Africains furent alors pris à partie parfois de manière violente. Pour apaiser les tensions, il fallut d'abord le concours d'une répression policière de grande ampleur qui n'eut d'autre effet que de liguer les rancœurs des communautés contre l'institution. Mais c'est surtout le rôle des leaders religieux du quartier qui fut déterminant. Sur la base d'un culte commun, les conflits cédèrent finalement la place à l'instauration d'un lien social. De cet apaisement, peuvent naître alors des passages de relais et des relations d'affaires qui assurent aux Africains subsahariens une légitimité de présence dans le quartier.

À Buenos Aires, I'histoire des relations inter-ethnique entre les Africains et les autres groupes de migrants n'a pas eu de débuts houleux comme à Marseille. Très rapidement, les commerçants sud-coréens, qui possèdent des bazars dans le quartier d'Once, comprennent qu'ils peuvent dégager de nouveaux profits en nouant des relations d'affaires avec leurs homologues africains. Si leurs bazars regorgent de marchandises diverses et variées, les Africains jettent leur dévolu sur la bijouterie-fantaisie, marchandise peu coûteuse, peu volumineuse et facile à écouler dans une organisation commerciale qui repose sur l'ambulantage. Par ailleurs, les Sénégalais, au début de leur présence en Argentine, pratiquent exclusivement le commerce à la valise et vendent leurs marchandises à l'extérieur d'Once. Les Coréens ont alors tout intérêt à ne pas s'opposer à l'ambulantage, puisqu'il se déroule hors de leurs espaces de vente. Au contraire, avec l'afflux des Sénégalais qui investissent en masse sur cette activité, les Coréens sont certains de faire croître leurs profits.

Dès lors, face à une demande accrue en bijoux-fantaisies, les Coréens concluent des accords avec les Africains qui induisent un nouvel ordre commercial. Les premiers cessent de vendre au détail les articles de bijouterie pour les affecter seulement à la vente en gros. Les seconds s'engagent à acheter les marchandises aux Sud-Coréens. Les Sénégalais, en contrepartie, ont le champ libre pour opérer comme détaillant. Certains Sénégalais, lassés des distances parcourues chaque jour avec leurs valises, envisagent de sédentariser leur activité. Le passage du colportage à la " sédentarité " ne traduit pas une progression économique. Selon les dires des enquêtés, les gains seraient plus ou moins identiques. L'acquisition d'un emplacement permet surtout de réduire considérablement la pénibilité du travail.

Pour mener à bien cette entreprise, ils obtiennent alors d'occuper un bout de trottoir, à proximité de la boutique de leur grossiste asiatique. En permettant aux 
migrants africains d'installer leurs étals à côté de leurs bazars, les Sud-Coréens favorisent alors progressivement l'ancrage commercial des Africains dans le quartier d'Once. Légitimés par ces passages de relai, les vendeurs de rue africains se rendent visibles des autres communautés commerçantes présentes sur le même espace marchand. Peu à peu, grâce à leurs facultés à tisser des liens et à se faire accepter en n'apparaissant pas comme des concurrents potentiels, ils négocient de nouveaux emplacements et s'imposent désormais, aux côtés des Péruviens, des Boliviens, ou encore des Paraguayens, comme des figures incontournables du commerce de rue à Buenos Aires et ailleurs en Argentine.

En dépit de ces passages de relai, des tensions peuvent aussi survenir. Les enjeux autour de l'appropriation de l'espace et la très grande densité du commerce de rue qui accroissent les mises en concurrence font parfois naître des frictions entre vendeurs à la sauvette.

El Hadj, originaire de la région de M'Bour au Sénégal, s'est ainsi heurté aux réticences d'un vendeur libérien qui ne voyait pas d'un bon œil l'installation de ce nouveau concurrent à quelques mètres seulement de son emplacement. El Hadj donne la version suivante:

"Lui [un vendeur libérien] est venu me voir en me disant qu'ici c'est pas possible, qu'on était déjà trop nombreux. Lui, il est fou et jaloux. Moi, je ne suis pas jaloux, quelqu'un peut travailler à côté de moi, ça ne me gêne pas. Ça ne me dérange pas, tout le monde a le droit de gagner son déjeuner, de payer son café. Regarde ici, on est trois Sénégalais et ça ne me dérange pas du tout ; lui, il est fou. Ces gens gaspillent tout leur argent, ils gaspillent 500 pesos dans un bar, ils invitent des filles et tout, et si tu leur demandes deux pesos, ils ne te donnent rien. Nous, les Sénégalais, on travaille, on partage, on s'aide, on se paye notre déjeuner et on envoie au pays. Au Sénégal, on est né en partageant, on partage tout sauf la femme " (El Hadj, conversation informelle, 20 août 2009).

Le discours du Sénégalais met en exergue un processus de glissement vers le facteur culturel pour expliquer les résistances du Libérien à son installation. El Hadj déplace ainsi la question d'un conflit sur l'occupation de l'espace entre deux individus, au terrain essentialiste qui consiste à opposer deux groupes distincts. Le passage soudain du " il " à " ces gens " exprime ce glissement vers une opposition entre " eux " et " nous ". II s'appuie alors sur un ensemble de valeurs positives de partage et de solidarité qui seraient ancrées dans la société sénégalaise pour mieux condamner des pratiques jugées déviantes et individualistes des "Autres ". L'instabilité, la précarité et la concentration de commerçants sur un espace restreint constituent un terreau favorable à l'expression des stéréotypes.

Cet exemple montre que le partage d'une expérience migratoire et d'une activité économique ne suffit pas à fédérer des groupes de migrants africains autour d'une même communauté de destin. Au contraire, les anglophones et les Sénégalais sont parfois des rivaux et mettent en scène des manières de se distinguer, notamment à travers les supports qu'ils utilisent, ou encore par le commerce à la valise, créneau occupé seulement par les mourides.

L'exemple d'un différend survenu entre deux vendeurs de rue de sousvêtements souligne bien la prééminence de la lutte pour l'appropriation des 
ressources sur d'éventuelles tensions identitaires. Suite à une saisie de sa marchandise par la police, une vendeuse péruvienne de l'avenue Rivadavia, exprime sa colère et son désarroi à un jeune sénégalais originaire de Diourbel: " II n'a pas sifflé ce connard, c'est un chien ! Maintenant, j'ai tout perdu, comment je vais nourrir mes enfants ? ", répète-t-elle plusieurs fois et à haute voix dans le but de désigner publiquement le présumé coupable, également de nationalité péruvienne. Elle accuse ce dernier d'avoir volontairement omis de l'avertir de l'arrivée de la police afin de précipiter sa faillite économique pour pouvoir récupérer sa clientèle.

Malgré cette intervention et en dépit du caractère illégal de la vente à la sauvette, la police se prête généralement à un jeu de dupe face à ces implantations informelles.

\section{La surveillance policière : un jeu de dupes}

Après avoir obtenu l'accord des autres commerçants, le futur vendeur de rue doit ensuite négocier le prix du "loyer " de son emplacement avec un policier en charge du secteur. Si les commerçants de rue ne payent pas de patente à la manière du commerçant établi, la rue a néanmoins un coût et c'est la police qui se charge de fixer les tarifs hebdomadaires selon la qualité de l'emplacement et selon la marchandise vendue. Ainsi, la vente de vêtements, qui requiert plus d'espace que la bijouterie, est supposée plus rentable, donc l'extorsion est plus élevée : en 2010, dans le quartier d'Once, elle s'élève à 170 pesos par semaine, contre quatre-vingt-dix, pour la bijouterie. À cette somme, s'ajoutent " des cadeaux " occasionnels. Afin d'éviter d'éventuelles représailles qui pourraient conduire à une confiscation de la marchandise ou à une expulsion du quartier, les commerçants se doivent d'accéder aux désirs des policiers, en leur donnant un produit qui figure sur leur étalage. Il convient de souligner que ces " dessous de table " ne garantissent pas une protection en cas de descente d'employés municipaux chargés de signifier l'illégalité de l'activité et de confisquer la marchandise. L'acte de corruption est donc une condition à l'installation, mais n'omet pas le caractère instable et précaire propre à la vente de rue.

Les forces de l'ordre qui accordent un "permis " de vente en fermant les yeux sur l'illégalité de cette activité doivent dans le même temps apparaître comme une garantie de l'application de la loi. Dans ce cadre, la police se charge de mettre en place un contrôle. Très régulièrement, des voitures de police circulent à une très faible allure avec des lumières clignotantes aux abords des trottoirs. L'idée est de parader, mais aussi de renvoyer une image menaçante en rappelant aux vendeurs que les policiers sont les garants de l'ordre et de la loi. Et l'objectif est atteint lorsque l'on observe le vent de panique que soulèvent ces approches calculées. Dès que la rumeur, fondée ou infondée et liée à une présence policière, envahit l'asphalte, les trottoirs ou allées piétonnes changent en quelques minutes de visage et redeviennent d'un seul coup la propriété des passants. Contraint de dissimuler hâtivement sa marchandise dans des sacs, des valises ou dans une cache, le vendeur s'efforce d'éliminer les traces de son activité. Transformée quotidiennement en espace marchand par les colporteurs, la rue redevient pour quelques minutes un simple lieu de circulation. 
Ces parades quasi quotidiennes dans les rues de Buenos Aires sont destinées à impressionner les travailleurs de la rue, mais aussi à s'afficher face à la population locale comme un acteur de répression. II s'agit là d'une mise en scène du contrôle soigneusement orchestrée par les autorités. D'un côté, il faut se montrer menaçant pour les migrants afin d'exercer une réelle domination en vue de les rançonner le cas échéant. De l'autre côté, il s'agit de faire appliquer une loi qui interdit la vente dans l'espace public et de rassurer des commerçants établis qui ne voient pas tous d'un bon œil l'installation de vendeurs de rue à proximité de leurs vitrines. Si les Coréens retirent des dividendes de cette présence, pour d'autres, elle génère une obstruction de la voie publique et fait ombrage à leurs boutiques. À cet égard, un commerçant de textile d'Once affirme :

" II y a de plus en plus de vendeurs sur le trottoir. Le problème, c'est pas qu'ils nous font de la concurrence, mais qu'ils occupent toujours plus d'espace, et ça c'est très ennuyant pour les clients. Nous, on peut pas les expulser et quand la police vient le faire, ils reviennent ensuite " (extrait d'une conversation informelle, 18 octobre 2011).

Pour le propriétaire d'une boutique de vêtements spécialisée en grande taille, la concentration de camelots dans le quartier est une forme de concurrence déloyale puisqu' ils ne payent pas d'impôts, pas de loyers et vendent à prix cassés ".

Ces expressions d'irritabilité amènent les élus locaux non seulement à formuler des discours qui condamnent cette pratique, mais aussi à entreprendre des actions spectaculaires. C'est dans ce cadre que le maire de Buenos Aires, Mauricio Macri ${ }^{12}$, élu depuis juin 2007 et par ailleurs leader du parti du centre droit Propuesta Republicana, avertit que :

"Personne ne peut s'approprier l'espace public. Les vendeurs à la sauvette doivent comprendre qu'ils agissent comme des hors la loi. L'espace public, c'est réservé aux gens qui marchent et qui veulent se promener, et pas pour que certains l'utilisent pour leurs propres bénéfices" (Territorio Digital, 18/01/2012).

Après avoir rappelé les normes qui régissent l'espace public, le maire menace alors les éventuels récalcitrants de faire appel aux forces de l'ordre pour les expulser. C'est d'ailleurs ce qui se produit en janvier 2012, lorsque près de 300 policiers sont réquisitionnés pour expulser les commerçants de rue qui travaillent sur I'avenue Corrientes (Territorio Digital, 18/01/2012).

En dépit des discours et de ce type d'action d'éclat, les patrouilles policières, si elles sont régulières, laissent tout de même une marge de manœuvre aux migrants dans leurs déplacements. En se rendant visible, à travers les véhicules et les sirènes sonores et illuminées, les policiers laissent généralement le temps au commerçant d'empaqueter son stock et de prendre la fuite. Dans une sorte de jeu de rôle, les policiers feignent l'arrestation en s'approchant suffisamment près des vendeurs à la sauvette pour que ceux-ci s'éloignent ou se cachent avant de revenir sur les lieux quelques minutes plus tard après le départ des voitures de police. Des logiques semblables ont pu être observées sur le parvis de la tour Eiffel, où les policiers ne déploient généralement pas tous les moyens disponibles pour confisquer la marchandise des camelots (François, 2004).

12 Désormais président de la Nation argentine depuis octobre 2015. 
C'est donc un jeu de surveillance relâchée ou molle qui met en lumière la situation ambiguë des forces de police. D'un côté, le policier est censé garantir le respect des lois, de l'autre, il ne désire pas tarir une source de rentrées financières.

Malgré l'importance du rôle des autorités policières, les règles qui président à l'accès au trottoir et à la légitimité de la présence restent une entreprise difficile à décoder ; si cette implantation revêt une "dimension hiérarchique ", les petits arrangements politiques et financiers ne peuvent expliquer in extenso "I'organisation sociale des lieux" (Sciardet, 2003). L'autre posture selon laquelle la rue serait soumise aux lois de la jungle et dépourvue de normes fabrique un aperçu éculé de cette scène urbaine.

\section{La rue comme vecteur d'alliances fluides}

Si la rue véhicule souvent des représentations de crainte, d'insécurité, elle génère cependant des pratiques de sociabilité et de convivialités le temps par exemple d'une emplette ou d'une pause-déjeuner. Espace d'ouverture, de mouvement, mais aussi de sédentarité, de brassage, de cohabitation et de libre circulation, la rue est le lieu de "côtoiement ethnique généralisé " (BordesBenayoun, 2005) ou encore le siège du cosmopolitisme. Hily et Rinaudo (2003) ont bien montré la manière dont le marché de Vintimille fabriquait des " formes originales de socialité " basée sur la fluidité des liens, où les alliances se font et se défont au gré des circonstances, où le proche et le lointain se confondent, où les rencontres fortuites peuvent étendre un capital social bien au-delà du réseau communautaire. La rue comme théâtre d'expression de "l'économie informelle mondialisée " (Hily et Rinaudo, 2003) apparaît également sous cette forme dans le quartier d'Once.

Ce ne sont pas seulement des échanges marchands qui organisent les rapports sociaux sur les trottoirs d'Once. C'est tout un quotidien à l'intérieur duquel se jouent de petites scènes sociales et les interactions que les Africains partagent avec des Argentins, des Péruviens, des Boliviens et quelques Brésiliens, vendeurs de rue également. Ce milieu de la vente ambulante, hétérogène de par les origines multiples des commerçants et des produits proposés, a toutefois en commun l'expérience de la migration et l'occupation de l'espace public. Se retrouver dans les mêmes lieux et pratiquer la même activité crée des liens de sociabilité. Ainsi, chaque jour, on se salue bien souvent comme les Argentins, c'est-à-dire en se faisant la bise indistinctement de son identité sexuelle, on s'efforce de prendre des nouvelles des uns et des autres, on évoque la vie du quartier, et les facteurs conjoncturels de la faiblesse des ventes telle que la pluie, la fin du mois, la crise, la grève dans les transports. Le commerce de rue ne se définit pas seulement par un amoncellement de diverses marchandises vendues sur un coin de trottoir ou à la sortie d'une bouche de métro. Au-delà des transactions et de la relation vendeur-client, $c^{\prime}$ est toute une vie de quartier qui se joue. Avec l'arrivée des vendeurs ambulants, l'espace public se mue en espace marchand qui prend la forme du marché et des sociabilités qui lui sont associées. À l'image du marché, l'oralité occupe une place déterminante dans les relations sociales. Ces acteurs mis en présence par le trottoir, le carrefour, la rue, l'avenue ou le quartier, échangent de la parole, des informations, de la confiance et plaisantent. 
On informe d'une éventuelle présence policière, on se prête de la monnaie en vue de rendre l'appoint au client. II est fréquent que des vendeurs du quartier s'arrêtent devant les échoppes des Africains pour leur demander comment se portent les affaires ou commenter la météo qui selon eux explique la forte ou au contraire la faible fréquentation.

À ces conversations à caractère amical, se superposent aussi des pratiques de solidarité. II est d'usage, par exemple, de confier son affaire à son voisin de trottoir si l'on est contraint de s'absenter quelques minutes, le temps d'aller faire un achat, de passer un coup de téléphone, ou de faire ses besoins dans les toilettes du bar ou du restaurant le plus proche, ou bien d'aller manger. Pendant ces absences, le plus proche confrère, indépendamment de sa nationalité et après avoir pris connaissance des prix des articles, se charge de "tenir la boutique ". Ces pratiques sont en réalité très répandues chez les vendeurs de rue.

Malgré une logique marchande qui répond à une démarche de profit le plus souvent individualisé ${ }^{13}$, les commerçants agissent comme s'ils étaient des associés ou des collègues de travail selon des liens de solidarité réciproques: outre les remplacements pendant les pauses, on s'entraide pour assembler les supports de vente ou, au contraire, pour les démonter après une journée de travail harassante, on se prête de la monnaie en cas de besoin, on recommande tel ou tel vendeur si on ne dispose pas de la marchandise réclamée par l'acheteur.

Loin des représentations de la grande ville, faisant de l'individualisme et de I'anonymat la base des relations sociales, ces exemples montrent au contraire que la rue peut initier des pratiques de convivialité et de solidarité dans un environnement d'interconnaissance. Ainsi, à l'image de nombreux Sénégalais, Dramane est connu et apprécié dans le quartier. Les commerçants du coin saluent ces qualités de gentillesse, d'amabilité et de courage, ce qui lui fait dire avec un certain orgueil :

"Ici, tout le monde me connaît, ça fait trois ans que je suis là, je n'ai jamais eu de problèmes avec personne et j'ai beaucoup d'amis ici ".

Kader témoigne des mêmes capacités à se "faire une place " au regard du grand nombre d'interactions interpersonnelles au cours desquelles on se salue, on demande des nouvelles de la famille ou d'un tel appartenant au réseau d'interconnaissance. En dépit d'une longue et épuisante journée de travail, le Sénégalais qui se fait aussi appeler "Pablo " prend encore le temps sur le chemin du retour de s'arrêter, de discuter, avec les uns et les autres, des rentrées financières, de la fréquentation et des événements qui rythment la journée, avant de leur souhaiter une bonne semaine.

Bamba mobilise également des compétences relationnelles et cosmopolites pour tisser des liens aussi bien avec des autochtones qu'avec d'autres migrants. Sa voisine commerçante est dominicaine et vend des survêtements. Quand les

13 Dans certaines communautés, comme chez les Boliviens ou les Péruviens, la vente est organisée par de petits collectifs familiaux. 
clients se font rares, ils tuent le temps en palabres. Ils évoquent avec nostalgie leurs pays d'origine. La jeune femme regrette les colmado (épicerie-buvette) de Saint-Domingue où elle aimait se rendre avec ses amis pour danser sur les rythmes de la bachata et du merengue ${ }^{14}$. Bamba, originaire de Pikine, évoque plutôt sa préférence pour des ambiances reggae de la capitale sénégalaise. Au-delà de ces échanges interculturels, ils partagent également des informations en lien avec la condition migratoire. Bamba montre ainsi beaucoup d'intérêt pour le récit de la voisine dominicaine qui se prévaut d'avoir contracté un mariage blanc contre une somme de 2000 pesos. Cette stratégie d'acquisition de la nationalité argentine est envisagée à des fins de réémigration. La jeune femme projette en effet de se rendre en Espagne, pays qui ne demande pas de visas aux Argentins, mais seulement un passeport valide. Bamba, souhaiterait visiblement imiter la jeune femme et $c^{\prime}$ est dans ce but précis qu'il lui demande alors si elle possède des "contacts ", c'est-à-dire des femmes argentines avec lesquelles il pourrait se marier. La Dominicaine répondra par la négative à ses attentes.

Par ailleurs, cette dernière se charge de surveiller et d'administrer le " bisness " du jeune Sénégalais quand il s'absente plusieurs fois par jour pour entretenir ses relations amicales. II accompagne épisodiquement dans les travées commerçantes d'Once des amies argentines venues y faire du " shopping ". II met alors à profit ces balades commerciales pour acheter sa marchandise dans les bazars des Sud-Coréens. Puis il s'arrête parfois chez des amies vendeuses qui travaillent dans une boutique de sous-vêtements pour prendre des nouvelles et discuter autour du maté ${ }^{15}$. Cette boisson a, dans la société argentine, une fonction de sociabilité et de convivialité. Le maté, qui peut se déguster en n'importe quel lieu à condition d'être équipé d'une bouteille isotherme pour conserver l'eau chaude, réunit parfois pendant de longues heures des convives qui peuvent alors s'abandonner au bavardage.

Nombreux sont les migrants africains qui se sont approprié cette coutume locale. Ils peuvent le consommer autant sur leur lieu de travail que dans les pensiones $^{16}$. Dans ces demeures, les Africains cohabitent très souvent avec des migrants latino-américains. C'est autour de la cuisine, pendant la préparation des repas, que les langues se délient. Youssouf, jeune Dakarois à qui, ce jour-là, revient la tâche de préparer le thiebou yapp ${ }^{17}$, accorde beaucoup d'attention au plat qu'une Péruvienne est en train de cuisiner. La Péruvienne explique alors

\section{Genres musicaux nés en République dominicaine.}

15 Cette infusion traditionnelle, à base de feuilles séchées de yerba-maté, se boit dans une gourde à maté et au moyen d'une paille en argent ou en simple métal. Consommé dans toutes les couches sociales, le maté se déguste selon un rituel qui consiste à faire circuler la gourde entre les convives dans le sens des aiguilles d'une montre alors qu'un cebador (" préparateur ") est chargé de verser régulièrement de l'eau chaude dans le maté.

16 Généralement de grandes bâtisses de trois ou quatre étages. Elles abritent de nombreuses chambres, une cuisine par étage, des toilettes et des douches communes, un patio, ainsi que plusieurs couloirs et escaliers qui en font de véritables labyrinthes.

L'état de ces vieux bâtiments présente de nombreuses imperfections; avaries de la tuyauterie, courts-circuits électriques, tapisseries et faux plafonds qui se décollent, meubles délabrés, caractérisent des conditions de logement médiocres.

17 Plat à base de riz et de viande (bœuf ou agneau) qui marine dans un assortiment de légumes. 
au Sénégalais la recette de l'escabèche qui consiste à faire mariner un poulet dans le vinaigre et la vapeur, avec des oignons. À la fin de la préparation, la jeune femme l'invite généreusement à goûter le met de son pays. Quelques instants plus tard, Youssouf, avec l'aide de deux de ses compatriotes, distribue le thiebou yapp dans les cinq chambres occupées par les Sénégalais. Dans l'une de ces chambres, un jeune Paraguayen d'une vingtaine d'années, qui vit à l'étage inférieur, s'est invité spontanément pour se délecter des saveurs sénégalaises en compagnie de ses "frères " africains selon ses propres termes. II n'en est visiblement pas à son premier essai ; accroupi par terre, il est en effet parfaitement à l'aise pour récolter avec sa main droite le riz servi dans un plat commun, conformément aux règles d'usage sénégalaises.

Ainsi, dans la rue ou dans les foyers, des sociabilités urbaines entre communautés de migrants s'organisent, pas seulement autour d'intérêts communs, mais aussi autour de ce que Simmel (1981) considère comme la forme la plus pure de la réalité sociale, au sens où les formes d'interactions qui caractérisent la sociabilité, ne se définissent pas par une fonction utilitariste, mais par :

"Une action réciproque des éléments par laquelle ceux-ci se constituent précisément en une unité ; et puisque (dans le cas de la sociabilité) les motivations concrètes de l'unification, rattachées aux finalités de la vie s'évanouissent, il faut accentuer avec d'autant plus de force et d'efficacité la pure forme, c'est-à-dire le lien de réciprocité, qui flotte en quelque sorte librement entre les individus " (Simmel, 1981 : 125).

Si Once se définit comme un espace d'insertion pour les communautés migrantes qui développent ensuite entre elles des formes plurielles de sociabilités, le quartier ne peut toutefois pas accueillir tous les candidats à la vente de rue.

\section{À la conquête des territoires}

Après avoir commencé à Once, les mourides explorent peu à peu d'autres territoires. La grande concentration de colporteurs et de vendeurs à la sauvette dans le quartier conjuguée à une accélération du flux d'entrée de Sénégalais créent une saturation progressive du marché et quelques tensions aussi entre les communautés. Des mourides continuent toutefois d'occuper Once, tandis que d'autres se lancent alors dans la conquête de nouveaux territoires. Cette conquête concerne d'abord les quartiers de la capitale qui ont des caractéristiques similaires à Once, en l'occurrence une concentration de population et la présence d'infrastructures de transport. Les gares de Constitución, au sud, et de Retiro, à l'est, ou encore Liniers, à l'ouest de la capitale argentine, donnent également lieu à une appropriation de l'espace par les vendeurs de rue. Ces implantations commerciales vont être reproduites dans les axes et les places secondaires de la capitale, puis dans les villes de banlieue du Grán Buenos Aires, comme à Morrón. Les mêmes logiques de négociation président à ces ancrages : respecter le principe de non-concurrence avec les autres commerçants, corrompre la police et faire marcher l'économie du lieu en consommant ou en versant par exemple une rétribution financière aux commerçants établis qui stockent le matériel et les marchandises.

Ainsi, la mobilité des vendeurs de rue est inhérente à ce type d'activité économique. Elle correspond à une adaptation aux logiques de mouvement des 
foules. Ces mouvements peuvent également de façon temporaire dépasser le cadre urbain. Pendant l'été austral, de la mi-décembre à la mi-février, les plages du littoral atlantique entre Mar del Plata et Carmen de Patagones accueillent des millions de vacanciers venus s'adonner aux plaisirs de la mer. Cette colonisation estivale représente pour les colporteurs mourides une opportunité unique d'écouler leurs marchandises. Sur des dizaines de kilomètres, et à la manière de leurs homologues sur la Costa del Sol (Espagne) ou sur la Côte d'Azur, les mourides bravent le soleil et la chaleur pour tirer profit de cette concentration humaine.

Cette concentration touristique entraîne ainsi dans son sillage un déplacement en masse des vendeurs ambulants qui, pour quelques semaines, abandonnent les rues de Buenos Aires et de sa périphérie.

Outre le redéploiement et la dispersion spatiale, la saturation progressive de I'activité conduit également certains commerçants à diversifier leurs stocks. Quelques Sénégalais s'essaient à la vente des casquettes et des lunettes de soleil. Sur l'avenue Corrientes, un autre Sénégalais propose des figurines de héros de dessins animés, mais aussi des gants et des bonnets pendant I'hiver. En d'autres termes, l'accroissement de la concurrence génère des initiatives individuelles qui, en affirmant leur distinction à l'égard du groupe, cherchent à exploiter de nouvelles niches économiques. Si, dans son étude sur les mourides de France, Salem (1981) avait souligné la prégnance de la contrainte dans l'organisation de la communauté, ces exemples de réorientation de la stratégie commerciale montrent au contraire une certaine liberté de mouvement. En France, les échanges avec le Sénégal comme base de l'organisation commerciale, participaient également au maintien du contrôle social. En Argentine, le caractère récent de la migration mouride, la distance et le coût du voyage ne favorisent pas la mise en œuvre d'échanges avec le Sénégal. En réponse à ces contraintes, les talibés exploitent plutôt le marché local.

Salem (1981) souligne également que "les alliances confrériques sont, le plus souvent, greffées sur les stratégies familiales ". En se superposant, le réseau familial et le réseau confrérique permettent de rendre avantageux un commerce fondé sur la multiplication des intermédiaires, des produits, des lieux d'approvisionnement et des lieux de vente pour pouvoir réaliser certaines marges. À Buenos Aires, la configuration est toute autre; la gamme de produits est peu élargie : des colliers, des bagues, des chaînes, des bracelets et des montres constituent le stock. Les lieux d'approvisionnement ne varient guère en dehors des fournisseurs sud-coréens. Le système commercial ne semble pas encore aussi complexe que celui détaillé par Salem.

Enfin, l'impossibilité de créer un monopole sur les produits mis en vente par les colporteurs peut constituer aussi un élément d'explication. Ce sont les Sud-coréens de Buenos Aires qui contrôlent les étapes de la commercialisation de la bijouterie-fantaisie fabriquée en Chine. À ce titre, Cohen (1971) avait souligné à propos de l'évolution du commerce en Afrique de l'Ouest :

"Lorsqu'un groupe ethnique est responsable de la plupart des étapes de commercialisation, il lui est plus facile de surmonter les difficultés de l'échange à distance, lequel s'applique également aux membres d'une confrérie islamique " (Cohen, 1971 : 266). 
C'est sans doute dans la capacité à contrôler une filière commerciale que se situe l'enjeu de la durabilité de la migration sénégalaise en Argentine. Un marché lié à la sous-culture afro en Argentine a commencé à être exploité par quelques commerçants récemment établis, mais ce n'est là qu'un préambule timide ; cependant, Bava (2002) a souligné que le dispositif mouride ne reposait pas uniquement sur la relation confrérique. À Marseille, des acteurs d'origine ethnique différente intervenaient dans l'organisation de l' "économie de bazar ". Malgré des conflits dans les années 1980 avec les Maghrébins, les commerçants mourides s'approvisionnaient grâce à des réseaux qui ne sont pas forcément communautaires et leur clientèle n'était pas sénégalaise. En ce sens, il ne s'agit pas d'un commerce ethnique, car toute la chaîne commerciale n'est pas maîtrisée par les mourides. On retrouve cette logique à Buenos Aires où les partenaires commerciaux des Africains peuvent être des Sud-Coréens concernant la bijouterie, des Péruviens quand il s'agit de s'approvisionner en paire de lunettes de soleil. La vente, comme à Marseille, n'est pas destinée à la communauté sénégalaise.

Par ailleurs, I'incapacité à connecter les territoires en tirant profit des différentiels de richesses, à l'inverse par exemple de certains Maghrébins de France qui multiplient les voyages entre leur terre d'accueil et celle d'origine, limite les " coups " et les opportunités commerciales. Ces migrants, que Tarrius (2002) appelle "fourmis ", construisent une " mondialisation par le bas " lorsqu'ils réussissent à élaborer des formes nomades de l'échange. II est évident que la comparaison n'est que très peu pertinente au regard de l'ancienneté des liens coloniaux et des transformations qu'ils ont induites à la fois dans la société française et dans les régions de départ. A contrario, l'Argentine se caractérise comme une nouvelle destination, lointaine tant du point de vue géographique que culturel. L'absence de liens entre le Sénégal et l'Argentine constitue certainement un handicap pour des commerçants qui souhaiteraient initier des échanges entre les deux pays. Cependant, les émigrations précédentes en Italie, en Allemagne, ou encore aux États-Unis ont mis en exergue la capacité des Sénégalais à organiser des réseaux et des circuits commerciaux sur des grandes distances ${ }^{18}$. II est donc permis de supposer que la jeunesse de cette migration explique également cette absence de pratiques économiques transnationales.

Dans l'évolution de ce dispositif commercial, il est également permis de postuler un renforcement des alliances avec les communautés boliviennes, paraguayennes ou encore péruviennes, nombreuses sur le sol argentin. C'est peut-être par l'intermédiaire de ces communautés, avec lesquelles les Sénégalais partagent le trottoir, que de nouveaux territoires vont s'ouvrir et faire naître des opérations commerciales. On peut alors légitimement se demander si ces inscriptions par le bas sur le territoire argentin sont de nature à durer ou peuvent déboucher sur des ascensions à l'image des mourides de New York. Ebin et Lake (1992) ont bien montré comment certains camelots, qui vendaient des parapluies ou des lunettes de soleil, sont ensuite sortis de la précarité grâce à la force des liens de solidarité communautaire pour connaître des réussites commerciales spectaculaires. En effet, ceux qui obtenaient les meilleurs gains mettaient en commun leur capital pour acheter la marchandise destinée à être

18 Voir les travaux de MBoup (2000) en Italie et Marfaing (2003) en Allemagne. 
revendue par les vendeurs ambulants. Grâce à ce marché rentable, ils sont devenus propriétaires de commerces de gros fréquentés également par les non-Sénégalais. Cette expansion s'est accentuée avec la transformation de leurs boutiques de gros en bureau d'import-export. Initialement colporteurs, ils se sont mués en acteurs économiques de premier plan. Ces mécanismes de l'ascension économique et sociale pourront-ils être imités en Argentine ou bien assistera-t-on à une stratégie de repli vers d'autres horizons ? Le développement dans les années à venir d'un type de commerce ethnique autour de l'africanité, encore phase d'émergence, et/ou l'essor d'un commerce transnational pourront vraisemblablement apporter des éléments de réponse.

\section{Références bibliographiques}

Ba Cheik Oumar et Ndiaye Alfred Iniss (2008) L'émigration clandestine sénégalaise, Revue Asylon(s), 3, [en ligne] consulté le 04/07/2015. URL : http://www. reseau-terra.eu/article717.html

Bava Sophie (2005) Variations autour de trois sites mourides dans la migration, Autrepart, 36, pp. 105-122.

Bava Sophie (2002) Routes migratoires et itinéraires religieux. Des pratiques religieuses des migrants sénégalais mourides entre Marseille et Touba, Thèse en anthropologie, EHESS, 625 p.

Bordes Benayoun Chantal (2005) De la rue ethnique au vaste monde, in Jeanne Brody, La Rue, Toulouse, Presses Universitaires du Mirail, pp. 281-292.

Bredeloup Sylvie (2008) L'aventurier, une figure de la migration africaine, Cahiers internationaux de sociologie, 125 (2), pp. 281-306.

Choplin Armelle (2010) Quand la mer se ferme. Du transit au post-transit en Mauritanie, Hommes et Migrations, 1286-1287, pp. 74-85.

Cohen Abner (1971) Cultural strategies in the organization of trading diasporas, in Claude Meillassoux, L'évolution du commerce en Afrique de l'Ouest, Oxford, Oxford University Press, pp. 263-281.

Colom-Jaén Artur (2012) La coopération Sud-Sud au Sénégal. De réelles opportunités de développement?, in Momar-Coumba Diop Dir., Sénégal (20002012). Le Sénégal sous Abdoulaye Wade. Le Sopi à I'épreuve du pouvoir, Paris, Karthala, pp. 765-792.

Contarino Sparta Luciana (1998) La comunidad caboverdeana en La Provincia de Buenos Aires: una historia ligada a la navegación, Revista de Historia Bonaerense, IV (16), pp. 49-51.

Copans Jean (2010) Espaces mourides, territoires étatiques sénégalais et mondiaux. Comment l'appartenance confrérique interpelle l'instance nationale, Islam et sociétés au sud du Sahara, 2, pp. 77-94.

Copans Jean (1980) Les marabouts de l'arachide, Paris, Le Sycomore, 263 p.

De Certeau Michel (1990) L'invention du quotidien. 1. Arts de faire, Paris, Gallimard, 349 p.

Dinh Bernard (2009) Un terrain marchand à l'épreuve de la diversité culturelle, Hommes et Migrations, 1280, pp $106-117$. 
Ebin Victoria et Lake Rose (1992) Camelots à New York, les pionniers de l'immigration sénégalaise, Hommes et Migrations, 1160, pp. 32-37.

François Sébastien (2004) Les vendeurs à la sauvette sur le parvis de la Tour Eiffel (observation), Terrains \& travaux, 7 (2), pp. 25-43.

Geerz Clifford (2003) Le souk de Sefrou : sur l'économie du bazar, Saint-Denis, Éditions Bouchène, $263 \mathrm{p}$.

Geerz Clifford (1998) La description dense, vers une théorie interprétative de la culture, Enquête, 6, pp. 73-105.

Germain Annick et Blanc Bernadette (1998) La vie de quartier dans le Montréal multiethnique, Revue Européenne des Migrations Internationales, 14 (1), pp. 141-160.

Hily Marie-Antoinette et Rinaudo Christian (2003) Cosmopolitisme et altérité : les nouveaux migrants dans l'économie informelle, Tsantsa (Revue suisse d'ethnologie), 8, pp. 48-57.

Koné Daouda (1995) Noirs-Africains et Maghrébins ensemble dans la ville, Revue Européenne de Migrations Internationales, 11 (1), pp. 99-114.

Levitt Theodore (1983) Globalization of Markets, Harvard Business Review, 61, pp. 92-102.

Lombard Jérôme et Steck Benjamin (2004) Quand le transport est d'abord un lieu, Autrepart, 32 (4), pp. 3-19.

Maffia Marta, Monkevicius Paola, Zubrzycki Bernarda, Agnelli Silvina y Ottenheimer Ana-Cristina (2012) Dinámicas asociativas entre los inmigrantes africanos subsaharianos en Argentina, in Mbuyi Kabunda Badi Dir., África en movimiento. Migraciones internas y externas, Madrid, Observatorio sobre la realidad social de Africa Subsaharian de la Fundación Carlos Amberes, pp. 1-24.

Marfaing Laurence (2003) Les Sénégalais en Allemagne, Paris, Karthala, 219 p.

Mboup Mourtala (2000) Les Sénégalais d'Italie. Émigrés, agents du changement social, Paris, L'Harmattan, $171 \mathrm{p}$.

Mera Carolina (2006) Migración coreana en Argentina, Nuevo Mundo Mundos Nuevos, [en línea] consultado el 10 septiembre 2014. URL: http://nuevomundo. revues.org/1286; DOI: 10.4000/nuevomundo.1286

Minvielle Régis (2015) L'Amérique latine ou I'expression d'une nouvelle route africaine, Afrique et Développement, XV (1), pp. 19-39.

Minvielle Régis (2013) Parcours africains en Amérique latine ou comment s'ébauche un dispositif migratoire transatlantique sud-sud, Thèse en sociologie, Aix Marseille Université.

Monnet Jérôme, Giglia Angela et Capron Guénola (2007) Ambulantage et services à la mobilité : les carrefours commerciaux à Mexico, Cybergéo, 371, [en ligne] consulté le 16/05/2013. URL : http://www.cybergeo.eu/index5574.html

Pliez Olivier (2012) Following the Silk Road between Yiwu and Cairo, in Gordon Mathews, Gustavo Lins Ribeiro and Carlos Alba Vega Dirs., Globalization from Below. The Word's Other Economy, Londres, Routledge, pp. 19-36.

Prahalad Coimbatore Krishnao (2004) The Fortune at the Bottom of the Pyramid. Eradicating Poverty Through Profits, Wharton School Publishing, 401 p. 
Raulin Anne (1988) Espaces marchands et concentration urbaine minoritaire : la Petite Asie de Paris, Cahiers Internationaux de Sociologie, 85, pp. 225-243.

Riccio Bruno (2006) "Transmigrants », mais pas " nomades ». Transnationalisme mouride en Italie, Les Cahiers d'études africaines, 181, pp. 95-114.

Salem Gérard (1981) De Dakar à Paris, des diasporas d'artisans et de commerçants. Étude socio-géographique du commerce sénégalais en France, Thèse, Paris, EHESS, $286 \mathrm{p}$.

Schmidt di Friedberg Ottavia (1993) L'immigration africaine en Italie : le cas sénégalais, Etudes internationales, 24 (1), pp. 125-140.

Sciardet Hervé (2003) Les marchands de l'aube : ethnographie et théorie du commerce aux puces de Saint-Ouen, Paris, Éditions economica, 217 p.

Simmel Georg (1984 [1979]) Digressions sur l'étranger, in Yves Grafmeyer et Isaac Joseph, L'Ecole de Chicago : Naissance de l'écologie urbaine, Paris, AubierMontaigne, pp 53-59.

Simmel Georg (1981) Sociologie et épistémologie, Paris, PUF, 238 p.

Tarrius Alain (2002) La Mondialisation par le bas : les Nouveaux Nomades de I'économie souterraine, Paris, Balland, 220 p.

Telles Vera (2009) Ilegalismos Urbanos e a Cidade, Novos estudos - CEBRAP, 84, pp. 153-173.

Timera Mahamet (2009) Aventuriers ou orphelins de la migration internationale ? Nouveaux et anciens migrants subsahariens au Maroc, Politique africaine, 115, pp. 175-195. 


\section{Régis Minvielle}

\section{Camelots sénégalais à Buenos Aires : une négociation cosmopolite du trottoir}

La récente migration africaine en Amérique latine s'inscrit dans un processus de reconfiguration du phénomène migratoire. Les parcours se déploient et se recomposent au gré non seulement de l'histoire des politiques migratoires, des crises et des opportunités économiques, mais aussi en fonction des histoires de groupes et $d^{\prime}$ individus. À Buenos Aires, la grande majorité développe une activité de commerce de rue sous l'impulsion notamment de la communauté mouride sénégalaise. Ces inscriptions par le bas s'effectuent en premier lieu dans le quartier central d'Once où se sont fondues au fil des époques des générations d'immigrés. De cet investissement de l'espace public, naissent des liens cosmopolites entre les différentes communautés de migrants qui envisagent la rue comme une ressource. Cependant, la saturation progressive de la vente ambulante à Once pousse les Sénégalais à explorer de nouveaux territoires.

\section{Senegalese Street Vendors in Buenos Aires: A Cosmopolitan Trading on Sidewalks}

African migration in Latin America is part of a process of reconfiguration of global migration. Paths unfold and recompose by the way of not only the history of migration policies, crises and economic opportunities but also by the groups and individuals stories. In Buenos Aires, the vast majority develops a street trading activity, driven mainly by the Murid Senegalese Community. These inscriptions from the bottom are made in the first place in the central neighborhood of the Once where generations of immigrants were implanted throughout the ages. From this investment of public space, cosmopolite links are born between various communities of migrants that consider the Street as a resource. However, the progressive saturation of Street vending in the Once leads the Senegalese to explore new territories.

\section{... Los vendedores ambulantes senegaleses en Buenos Aires: una negociación cosmopolita de la acera}

Las migraciones africanas en América Latina forman parte de un proceso de reconfiguración global del fenómeno migratorio. Los recorridos se despliegan y se recomponen al ritmo, no solo de la historia de las políticas migratorias, las crisis y las oportunidades económicas, sino también en función de las historias de grupos e individuos. En Buenos Aires, la gran mayoría desarrolla una actividad de comercio callejero, iniciada principalmente por la comunidad murid senegalesa. Estas inscripciones desde abajo se realizan en primer lugar en el barrio central de Once donde se implantaron a lo largo del tiempo generaciones de inmigrantes. De esta inversión del espacio público, nacen lazos cosmopolitas entre distintas comunidades de migrantes que consideran la calle como un recurso. Sin embargo, la saturación progresiva de la venta ambulante en Once Ileva los Senegaleses a explorar nuevos territorios. 